\title{
Tarifverträge und Vereinbarungen zwischen santésuisse und der FMH
}

La version française suivra
Die Einführung von TARMED auf den 1. Januar 2004 im KVG-Bereich verlangte nach den verschiedensten Begleitmassnahmen. Die G7 hat deshalb im vergangenen Jahr intensive Verhandlungen mit santésuisse geführt, um die noch offenen Punkte zu klären. Die Verhandlungen fanden in einem sehr konstruktiven, problem- und lösungsorientierten Klima statt. Dadurch konnten nicht nur alle kantonalen Ärztegesellschaften mit santésuisse in den Vertragsverhandlungen einvernehmlich die Starttaxpunktwerte berechnen, sondern es konnten auch die verschiedensten noch anstehenden Probleme einer Lösung zugeführt werden.

Folgende Probleme galt es auf das Einführungsdatum hin zu lösen.

\section{Vertrag über die Verrechnung von paramedizinischen Leistungen in der Arztpraxis}

(Physiotherapie, Ergotherapie, Logopädie, Ernährungs- und Diabetesberatung durch in der Arztpraxis angestelltes Fachpersonal)

Wer in der Praxis die entsprechenden Fachpersonen angestellt hat, kann diese mit dem ebenfalls beiliegenden Formular an santésuisse melden und ist gehalten, bei der FMH eine entsprechende EAN-Nummer zu beantragen.

Die ausführlichen Tarifwerke können bei santésuisse bezogen werden oder sind im Internet abrufbar.

Wer in der Arztpraxis lediglich Elektro-, Wärme- oder Kältebehandlungen durch die MPA durchführen lässt, rechnet diese über die Position 7320 (PhysioTarif) mit 10 TP ab. Die im Kanton gültigen TPW für die paramedizinischen Leistungen entnehmen Sie der publizierten Tabelle. Es ist nicht der TARMED-TPW zu verwenden.

\section{Gefährdete Fachgruppen}

TARMED könnte für einige Fachgruppen tarifbedingt zu erheblichen Einkommensproblemen führen, andere könnten ungewollt mit ihrem Abrechnungsvolumen stark überschiessen.

Um hier gezielt Massnahmen treffen zu können, haben FMH und santésuisse für den Bereich der niedergelassenen Ärzte eine Instanz geschaffen, welche gezielte Korrekturmassnahmen z.H. des Kostenneutralitätsbüros erarbeitet.

Sie sind hier alle gefordert, Ihren Fach- und Kantonalgesellschaften das notwendige Zahlenmaterial zur Verfügung zu stellen, um die notwendigen Korrekturen zu erarbeiten.

Voraussehbar absolut existentiell gefährdet sind durch die Einführung von TARMED die Radiologieinstitute. Dies zeigen auch alle bisherigen Erfahrungen aus dem UVG-Bereich.

Mit santésuisse zusammen konnten Notmassnahmen für die Radiologie errechnet werden sie treten per 1. Februar 2004 in Kraft.

\section{Vertreter der FMH in den Steuerungs- gremien der Kostenneutralität}

\section{Kostenneutralitätsbüro}

- Dr. Urs Stoffel, Präsident Zürcher Ärztegesellschaft;

- Dr. Charles A. Favrod-Coune, Präsident Société Médicale Vaudoise.

\section{Paritätisches Vorprüfungsbüro}

- Dr. Andreas Haefeli, Präsident Aargauischer Ärzteverband;

- Dr. Markus Wili, Präsident Luzerner Ärztegesellschaft;

- Dr. Jürg Pellaton, Zürcher Ärztegesellschaft.

Die Vertreter werden fachtechnisch und statistisch beraten durch ein Controllingteam der NewIndex AG. Die Verbindung zu TARMEDsuisse wird sichergestellt durch Jacques-Henri Weidmann, Chef Tarifdienst FMH.

Zusammen mit den Vertretern von santésuisse werden wir die Entscheidträger in zwei «Stabsübungen» anhand reeller Zahlen der Jahre 2002 und 2003 auf ihre Aufgaben vorbereiten.

\section{Rechnungsstellung}

Partnerschaftlich konnten die Formate der TARMED-Rechnungen von den Anforderungen des UVG-Bereichs auf die Anforderungen des KVGs und weiterer Gesetze (z.B. Mehrwertsteuergesetz) adaptiert werden. Die entsprechenden Un- 
